\title{
A Tecnologia da Informação na Área Hospitalar: um Caso de Implementação de um Sistema de Registro de Pacientes
}

\author{
José Rodrigues Filho \\ Jefferson Colombo B. Xavier \\ Ana Lívia Adriano
}

\section{ResUMo}

O sistema de registro médico computadorizado, ou seja, o desenvolvimento do prontuário eletrônico, é um dos temas mais discutidos nos dias de hoje na área de informática médica. Há afirmações de que a combinação de computadores, redes de telecomunicações, informações médicas e dados eletrônicos de pacientes pode melhorar a qualidade do cuidado de saúde, a produtividade dos profissionais de saúde, facilitar o acesso aos serviços disponíveis e reduzir custos administrativos associados à prestação de serviços. O registro de pacientes é considerado o módulo básico de um sistema computadorizado de gerenciamento de pacientes e, conseqüentemente, do prontuário eletrônico. Uma tentativa é feita, neste trabalho, para descrever a implementação de um sistema de registro de pacientes, denominado R-ATA (registro-admissão, transferência e alta), no Hospital Universitário Lauro Wanderley da Universidade Federal da Paraíba.

Palavras-chaves: registro de pacientes; prontuário eletrônico; registro-admissão e transferência; desenvolvimento de sistemas de informação.

\begin{abstract}
The computer-based patient record (electronic patient record) is an area of medical informatic that has been well discussed during this decade. It has been alleged that the powerful combination of computers, communication networks, online medical information, and electronic patient data can improve the quality of health services, increase access to health care, productivity of health professionals, and reduce unnecessary cost. Patient registration: admission/transfer/discharge is the basic module of a patient management system. The purpose of this work is to describe the implementation of a patient registration system, named R-ATA, implemented in the Lauro Wanderley Teaching Hospital of the Universidade Federal da Paraiba, Brazil.
\end{abstract}

Key words: patient registration; electronic medical record; admission/transfer/discharge; information system development. 


\section{INTRODUÇÃO}

Tem sido afirmado que a combinação de computadores, redes de telecomunicações, informações médicas online e dados eletrônicos de pacientes pode melhorar a qualidade e as decisões inerentes ao cuidado de saúde, além de facilitar o acesso aos serviços disponíveis. Assim sendo, tem-se enfatizado a automação do prontuário do paciente, já que os sistemas de arquivo médico baseados em computadores, por mais incompletos que sejam, contribuem significativamente para melhorar a qualidade do tratamento e o controle dos custos de saúde (Lindberg e Humphreys, 1995; Rodrigues Filho, 1995).

Não há dúvidas de que as tecnologias de informação e comunicação estão nos conduzindo a uma nova era, à medida que nos aproximamos do final do século XX; contudo não podemos deixar de nos preocupar com os limites da difusão dessas tecnologias, sobretudo na área de saúde: será que todos se beneficiarão do aumento das disponibilidades de recursos de saúde online, ou estamos caminhando para uma sociedade dos que têm e dos que não têm informações? (Thomas et al., 1998).

A disponibilidade da opção tecnológica que visa a uma combinação poderosa da tecnologia digital e dados é muito recente, razão pela qual os sistemas de informações clínicas desenvolvidos nos últimos vinte anos são considerados pobres em qualidade e desempenho, além de oferecerem poucas opções aos profissionais médicos (Chu, 1993).

A mais recente investida da informática médica está sendo direcionada ao desenvolvimento de sistemas de arquivos médicos computadorizados, a exemplo do projeto de informática médica, desenvolvido nos Estados Unidos, que objetiva automatizar o prontuário do paciente (Stead, 1989; Dick, 1991).

Um sistema de arquivo médico é um conjunto de componentes que forma os mecanismos para que os prontuários sejam criados, usados, armazenados e acessados, fazendo parte de um sistema de informação hospitalar (SIH) e tendo como foco central os dados clínicos.

Em 1989, por exemplo, o Instituto de Medicina da Academia de Ciências dos Estados Unidos formou uma comissão, composta por mais de duzentos membros representantes das instituições de saúde americanas, para estudar como a tecnologia poderia melhorar os sistemas de registro médico. Após dezoito meses de trabalho, a mencionada comissão constatou que tais sistemas não se diferen- 
ciavam daqueles existentes há cinqüenta anos, ou seja, a pobreza dos sistemas de arquivo médico já estava comprometendo a prestação do cuidado de saúde, já que não atendia às necessidades dos profissionais de saúde, pacientes, administradores e pesquisadores, apesar dos avanços tecnológicos na área de saúde durante as últimas décadas. Conseqüentemente, foi proposto um sistema de registro médico baseado em computador, ou seja, o desenvolvimento do prontuário eletrônico (Dick, 1991).

As barreiras apontadas, na época, para dificultar o desenvolvimento do prontuário eletrônico diziam respeito ao desenvolvimento de padrões, sobretudo na área médica, educação e treinamento dos usuários e desenvolvedores de sistemas, além de outros desafios técnicos e organizacionais.

A previsão do Instituto de Medicina americano era de que, dentro de uma década, ou seja, a partir do ano 2001, os objetivos de se automatizar o prontuário do paciente seriam atingidos. Diante das barreiras, já mencionadas, mas que ainda hoje existem, alguns anos serão necessários para se ver o prontuário eletrônico substituir o prontuário de papel, sobretudo nos países em desenvolvimento.

Embora se reconheça a necessidade de se melhorar os sistemas de informação em saúde e de ter havido avanço tecnológico e melhor padronização dos dados, ainda persistem a falta de consenso sobre conteúdos, desagregação do ambiente organizacional dos serviços de saúde, comportamento imprevisível do usuário, além de uma variedade de questões legais e sociais que precisam ser encaradas, a exemplo das políticas públicas, destinadas a disciplinar e facilitar o acesso dos cidadãos às redes de informações, evitando uma elitização e desigualdade social entre os que têm e os que não têm informações.

Os sistemas de prontuários eletrônicos requerem certo grau de precisão, o que não faz parte das práticas atuais, principalmente no Brasil. Os serviços de prontuários na maioria dos hospitais brasileiros, quando existem, são de baixo padrão, diante da desordem organizacional dessas instituições; portanto o serviço de arquivo médico, considerado o coração de qualquer instituição hospitalar, funciona de forma muito precária na maioria dos nossos hospitais.

Acontece que a documentação sobre os cuidados do paciente está se tornando imperativa, não sendo mais possível tolerar registros pobres, incompletos e desordenados, como os que existem na generalidade dos hospitais. O grande problema para se automatizar parte das atividades do serviço de arquivo médico não diz mais respeito aos custos, mas à educação dos profissionais de saúde, que interagem com o prontuário do paciente: médicos, técnicos, enfermeiros, entre outras profissões. 
Além disto, no Brasil, muitos dos sistemas de informações no setor saúde em geral, e hospitalar em particular, foram desenvolvidos sem nenhuma forma de padronização da informação, conforme critérios desenvolvidos por instituições nacionais ou internacionais. Assim, muitas vezes, dados sem utilidade são coletados, enquanto outros de maior importância deixam de ser registrados.

A padronização da informação exige grande esforço dos profissionais de saúde, sendo uma esperança para o controle de grande quantidade de informações clínicas e de pesquisa, que pode economizar o seu tempo valioso; portanto, considerando o desenho modular para o desenvolvimento de sistemas de informações hospitalares, o propósito deste trabalho é descrever a implementação de um sistema de registro de pacientes, denominado R-ATA (registro-admissão, transferência e alta), no Hospital Universitário Lauro Wanderley da Universidade Federal da Paraíba, o qual foi desenvolvido cumprindo padrões internacionais, como os especificados pela American Society for Testing and Materials (ASTM), que tem tratado da padronização da informação em saúde há mais de vinte anos (Megargle, 1991). Esta aplicação computacional poderá ser implementada em quaisquer hospitais, postos de saúde ou ambulatórios.

\section{Sistema de InformaÇÃo Hospitalar}

Um sistema de informação hospitalar integrado, como qualquer outro de área distinta, consiste em dois componentes: hardware e software. Contém diversos módulos ou subsistemas, dentro dos seguintes grupos funcionais: administração, gerenciamento de pacientes, aplicações médicas e sistema médico-técnico (vide Figura 1).

Historicamente, os sistemas de informações hospitalares foram desenvolvidos para atender inicialmente às necessidades administrativas: aplicações financeiras, folha de pagamento, contabilidade etc. Em seguida enfatizou-se a automação dos sistemas médico-técnicos: patologia, radiologia, laboratórios, farmácia etc., os quais compõem também um sistema de informação hospitalar.

A tecnologia da época facilitou automatizar sistemas alimentados por dados ou informações bem estruturadas, a exemplo dos sistemas administrativos e médicotécnicos. As informações necessárias ao cuidado direto do paciente diferenciam-se qualitativamente das informações que apóiam as funções administrativas. Além disto, informações administrativas podem ser coletadas retrospectivamente, mas as informações sobre o tratamento do paciente devem ser oportunas e disponíveis no ponto ou hora do cuidado. 


\section{Figura 1: Sistema de Informação Hospitalar Integrado}

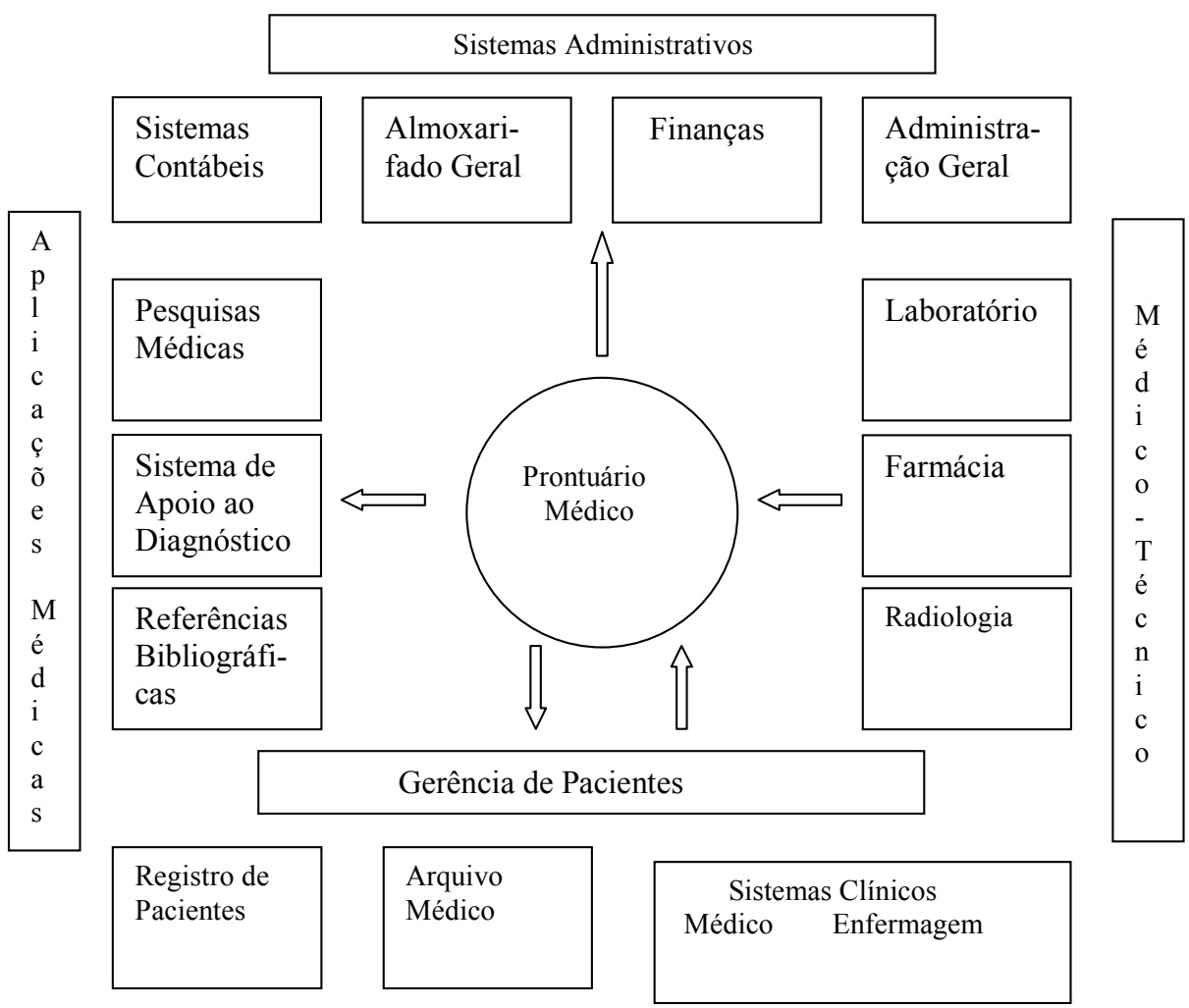

Assim, durante longo período, os hospitais gastaram muito em sistemas que nem sempre processavam informações inerentes às funções do cuidado do paciente, priorizando apenas as aplicações computacionais na área administrativa. Os sistemas de informações clínicas requerem considerações mais complexas de desenho do que os sistemas de informações administrativas.

Embora os preços do mercado de informática tenham caído substancialmente nos últimos anos, o software não tem acompanhado o desenvolvimento do hardware, sobretudo na área de saúde. Muitos dos sistemas de informações estão em desuso, pelo menos nos países desenvolvidos, já que foram adquiridos de diferentes fornecedores, impossibilitando a integração e a interação dos seus componentes.

Há comentários de que os sistemas de informações hospitalares demonstrados no ano de 1990, por exemplo, se pareciam com aqueles demonstrados no ano de 1975 (Ball et al., 1991), ou seja, poucas mudanças foram implementadas num período de quinze anos, apesar do grande avanço da tecnologia. 
A alegação era de que os vendedores de software estavam ainda tentando recuperar os seus investimentos destinados ao desenvolvimento de aplicações de software de épocas passadas. Infelizmente, no mundo ocidental, os vendedores de software e alguns consultores é que definem as necessidades dos usuários no tocante à definição de sistemas de informação. Conseqüentemente, a tecnologia da informação é orientada pelo mercado e não pelas necessidades do usuário, ou seja, as pessoas têm de se ajustar à tecnologia e não a tecnologia ser ajustada aos interesses do usuário, em benefício do bem-estar e qualidade de vida. Além do mais, muitas vezes quando a tecnologia da informação é introduzida nos países em desenvolvimento, tipicamente tem sido na forma de aplicações e sistemas que são "soluções do primeiro mundo" para "problemas do primeiro mundo" (Braa, 1996).

No caso específico da saúde, é preciso que os profissionais de saúde e as associações médicas e paramédicas comecem a ter papel decisivo na definição dos sistemas de informação, enquanto usuários, propondo até mesmo uma mudança de paradigma, quando necessário, em matéria de desenvolvimento de sistemas (Rodrigues Filho, 1999).

É comum encontrar instituições hospitalares com sistemas de informações financeiras, contas hospitalares, folhas de pagamento e muitas outras funções, que não atendem às exigências do hospital, por serem completamente desintegradas. Nos últimos anos, as organizações hospitalares têm-se preocupado com a integração de dados administrativos e dados clínicos, como forma desejável e necessária ao seu bom funcionamento.

Em 1988, a Associação Internacional de Informática Médica (International Medical Information Association - IMIA) fez a recomendação de que o tratamento do paciente se tornasse o foco principal dos sistemas de informações hospitalares (Ball et al., 1991).

Para alguns, a falta de sucesso dos sistemas de informações hospitalares pode ser atribuída à falha dos planejadores de sistemas em entender o fluxo de dados clínicos; à noção errada dos administradores de acharem que as aplicações médicas são um produto das aplicações administrativas; à falha de não incluírem os usuários, sobretudo os profissionais médicos, no desenho e implementação dos sistemas de informações hospitalares, além da falta de recursos destinados ao processamento automatizado dos hospitais (Friedman e Martin, 1997).

Os sistemas de gerenciamento de pacientes, considerados como nova geração de sistemas a integrarem um sistema de informação hospitalar, são mais recentes e tiveram origem no desenvolvimento de sistemas de registro de pacientes. Sistemas de informações hospitalares complexos e reconhecidos como o DHCP 
(Decentralized Hospital Computer Program), hoje instalado em dezenas de unidades de saúde da Administração dos Veteranos e do Departamento de Defesa dos Estados Unidos, tiveram como módulo básico e inicial o sistema de registro de pacientes (Munnecke e Kuhn, 1989). O mesmo aconteceu com o DIOGENE (Division d'Informatique Hôpital Genève), sistema de informação complexo, instalado num hospital de 1.600 leitos, na Universidade de Genebra (Scherrer et al., 1990). Merece destaque, também, o famoso sistema de informação COSTAR (The Computer Stored Ambulatory Record), desenvolvido por Barnett e seus colegas no Laboratório de Ciências da Computação do Hospital Geral de Massachusetts, e que tem como fundação o sistema de registro de pacientes (Blum, 1986).

A gerência de pacientes, como se vê na Figura 1, inclui sistemas de registro de pacientes e os denominados sistemas de arquivo médico e de informações clínicas, que agregam tanto as informações das atividades médicas quanto as das atividades de enfermagem (planos de tratamento, diagnósticos, ordens médicas etc), além de informações pessoais e demográficas dos pacientes.

Funcionalmente, a unidade de registro e admissão de pacientes faz parte do Serviço de Arquivo Médico e Estatística (SAME) de um hospital, sendo a fonte principal de informações pessoais do paciente. O arquivo médico, por sua vez, é considerado "o repositório centralizado de todas as informações pessoais e médicas sobre os pacientes de uma organização de atenção à saúde", tendo assim uma importância fundamental em praticamente todas as atividades clínicas e administrativas (Rodrigues, 1987). Com o advento da moderna tecnologia, tornou-se possível a automação de muitas das funções do SAME, incluindo as funções de registro de pacientes.

\section{Sistema de Registro de Pacientes}

Um sistema de registro de pacientes, denominado R-ATA (registro-admissão, transferência e alta), foi desenvolvido como módulo básico de um sistema de gerenciamento de pacientes, com as possibilidades de interligação com outros módulos, como se mostra na Figura 1. Esta aplicação computacional tem as seguintes funções:

- Identficar ou verificar pacientes por meio de um índice de pacientes criado e mantido pelo processo de registro. $\mathrm{O}$ índice de pacientes deve ser mantido não só por hospitais, mas por qualquer unidade de assistência médica, sendo uma subfunção do registro. 
- Manter o arquivo de registro e dados demográficos como um nó comum para o sistema de arquivo médico, de modo que possa ser utilizado por outros sistemas a serem desenvolvidos.

- Estabelecer o registro inicial de entrada de dados dos pacientes mediante a criação do segmento de dados demográficos, que serão usados para a criação de um índice de pacientes para o arquivo médico.

. Acompanhar a movimentação do paciente dentro do hospital.

. Produzir o censo de pacientes e relatórios estatísticos.

- Oferecer aos outros departamentos do hospital informações comuns sobre cada paciente registrado/admitido.

- Criar relatórios administrativos sobre a taxa de ocupação e média de permanência do hospital. O controle de leitos é a ferramenta gerencial que visa a assegurar que o leito, como fonte produtora de receitas, está sendo utilizado de forma eficiente.

- Identificar os médicos de cada paciente.

- Elaborar lista de altas hospitalares de interesse dos setores de admissão do serviço de arquivo médico, farmácia e outros departamentos.

Os desafios do gerenciamento das informações de saúde, em geral, e hospitalar em particular, não dependem apenas dos avanços tecnológicos. Além da tecnologia, um estudo da melhoria do registro de pacientes deve considerar como o uso desses registros deve ser melhorado. A melhoria dos registros e a racionalidade clínica dos profissionais de saúde são tópicos que estão inevitavelmente associados, uma vez que os registros médicos refletem o processo racional clínico. Se se deseja criar melhores sistemas de registro de pacientes, o usuário deve ser reconhecido e as atividades inerentes às soluções dos problemas dos profissionais de saúde devem ser examinadas.

Se os sistemas de arquivo médico nos hospitais brasileiros são pobres e desorganizados, o mesmo acontece em muitos hospitais de países desenvolvidos. O redesenho desses sistemas oferece o mais simples e imediato enfoque para o controle dos custos de saúde e a prevenção de erros médicos (Brunum, 1989). Há afirmações de que nos Estados Unidos os sistemas de registro médico são igualmente de má qualidade e perigosos, não contribuindo para a melhoria dos cuidados de saúde (Hirshey et al., 1989; Pories, 1990). Resumidamente, as principais falhas dos registros médicos podem ser descritas como sendo de conteúdo, formato, acesso e disponibilidades. 


\section{Problemas de Conteúdo e Formato}

Os dados do prontuário médico são freqüentemente ilegíveis, não acurados, fragmentados, incompletos, incompreensíveis para o paciente e seus familiares e, algumas vezes, excessivos ou redundantes. Nem sempre a documentação do prontuário é ordenada de forma lógica; a pobreza do seu formato impede uma utilização mais eficiente.

\section{Problemas de Acesso e Disponibilidade}

Já foi registrado que é grande o percentual de prontuários não localizados, ou seja, em 30\% das buscas nem sempre se localiza o prontuário (GAO, 1991). A indisponibilidade do prontuário cria sérios problemas e frustrações para o usuário. É prática comum o médico deixar o prontuário em diferentes setores do hospital e até em casa.

Tais informações são suficientes para mostrar quanto outros fatores dificultam o trabalho de automação do prontuário médico e quanto é importante educar os profissionais de saúde para lidar com a tecnologia da informação, sobretudo os que interagem com o prontuário médico.

Embora durante o desenvolvimento do R-ATA diversas questões referentes à padronização dos dados contidos nos sistemas de registro de pacientes, conforme especificações da ASTM, instituição envolvida com o desenvolvimento de padrões para as aplicações computacionais em medicina (Megargle, 1991), tenham sido consideradas, as questões inerentes ao conteúdo, formato, acesso e disponibilidade do prontuário não foram examinadas, além de outras questões de ordem organizacional e social.

Como veremos a seguir, isto dificultou a implementação de um sistema que, embora tecnicamente apropriado e justificado, estava predisposto à falhas causadas pela falta de especificação do grupo destinado a usá-lo e de um ambiente familiarizado com a tecnologia da informação.

Nenhuma tentativa será feita neste trabalho para revisar a literatura sobre o sucesso e falhas de implementação de sistemas de informação (Markus, 1983; Davis et al., 1992; Pollalis e Frieze, 1993), apesar de se reconhecer que este tópico é quase inexistente na literatura brasileira. 


\section{Metodologia de Implementação e Utilização do R-ATA}

O Hospital Universitário Lauro Wanderley dispõe de aproximadamente 250 leitos. Considerando a falta de recursos computacionais (hardware) do hospital, o sistema de registro de pacientes foi testado na Unidade de Clínica Médica, que dispõe de 70 leitos e é dividida em duas alas, comportando as várias especialidades médicas.

O ponto de partida para se iniciar o processo de armazenamento de dados no R-ATA se dá com o cadastramento dos prontuários, como exemplificado na Tela 1 (primeiro atendimento). Complementando as informações do primeiro atendimento, pode-se armazenar outros dados sociodemográficos, oriundos da ficha social preenchida pelos assistentes sociais do hospital, mediante entrevista. Estes dados são capturados tanto antes como depois da internação do paciente.

\section{Tela 1}

QP R I M E I R 0
A T E N D I M E N T O
PRONTUÁRIO

O gerenciamento das internações hospitalares inicia-se com o preenchimento da guia de internamento, que identifica quando e onde o paciente está internado, além de identificar a clínica e o médico responsável pela internação (vide Tela 2). 
Tela 2

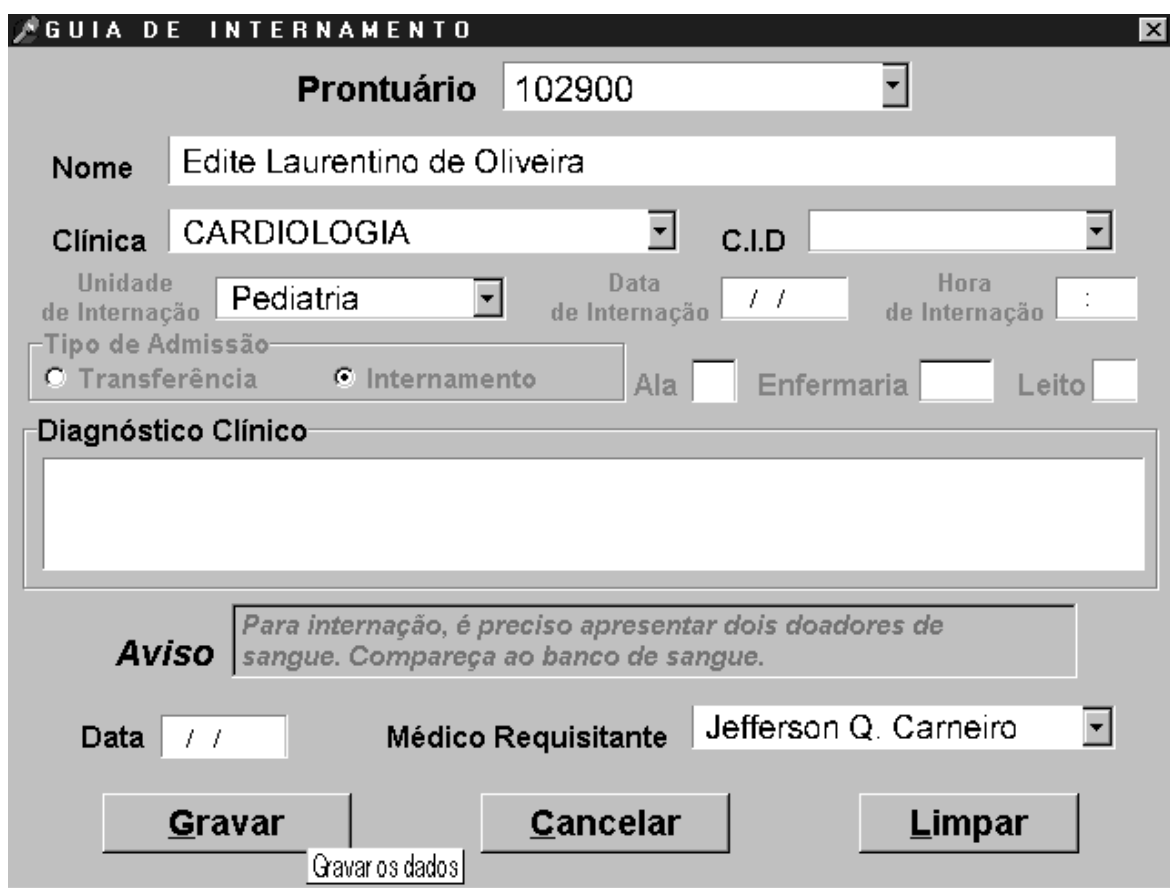

Inicialmente foi feito todo um esforço para capturar e registrar informações de todos os pacientes internados nas duas alas da Unidade de Clínica Médica, dispondo-se apenas de uma máquina (microcomputador). Percebeu-se, posteriormente, certo atraso no preenchimento de determinados documentos, principalmente da ficha social, preenchida pelas assistentes sociais do hospital. Desta feita, tornava-se impossível manter o sistema atualizado diariamente. Assim, e considerando a falta de recursos computacionais, optou-se pela implementação do sistema em uma única ala, abrangendo 35 leitos. Neste caso, foi possível manter o sistema atualizado diariamente.

Em geral, a internação é feita após o preenchimento da ficha social do paciente; contudo pacientes internados nos finais de semana ou dias feriados só têm a sua ficha social preenchida posteriormente, ou seja, dois ou três dias depois. Como já foi dito, informações sobre o tratamento médico não devem ser coletadas retrospectivamente, mas devem ser oportunas e disponíveis no ponto e na hora do cuidado.

Várias outras telas vão sendo simultânea e automaticamente preenchidas, a partir do primeiro atendimento e do processo de internação do paciente: gerência de leitos, cadastros das clínicas, médicos etc. Diversos relatórios são produzidos 
pelo sistema, a exemplo do relatório de ocorrências (vide Tela 3) e outros relacionados com importantes indicadores de produtividade hospitalar: taxa de ocupação hospitalar, pacientes-dia, média de permanência etc.

\section{Tela 3}

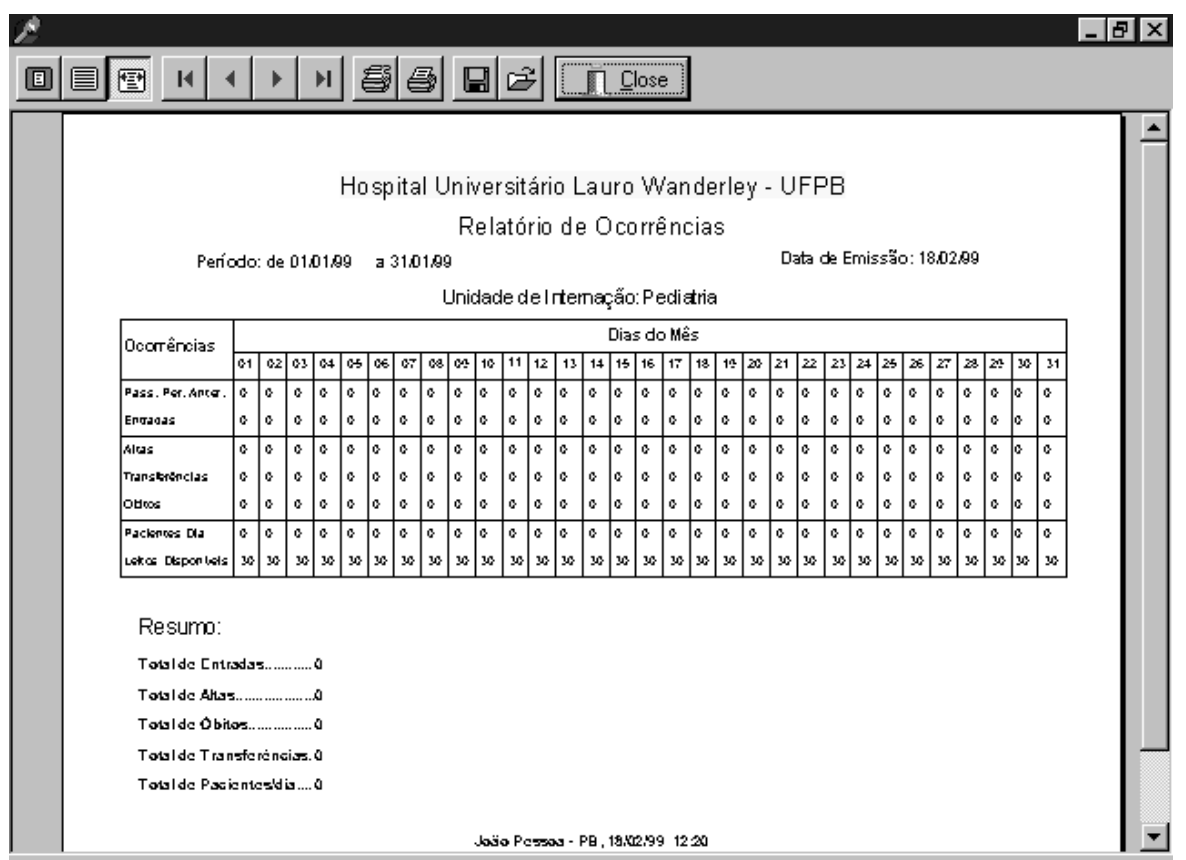

Page 1 of 1

As funções de alguns desses relatórios e as suas respectivas alterações discriminam-se a seguir.

Relatório de ocorrências por unidade: trata-se de relatório que fornece a quantidade de altas, internações, transferências, óbitos e pacientes que passaram do dia anterior, a cada dia do mês.

Mapa diário de internação: relatório que reflete a ocupação atual dos leitos masculinos e femininos por especialidade médica.

- Resumo geral da entrevista social: relatório que fornece a quantidade detalhada de cada item da entrevista social realizada no período fornecido pelo usuário.

- Gerência de leitos: relatório que permite à administração de enfermagem visualizar graficamente a ocupação dos leitos em cada ala, especificando os pacientes internados em determinada enfermaria ou leito. 
. Cadastro de pacientes: permite corrigir ou acrescentar informações dos prontuários dos pacientes.

. Entrevista social do paciente: permite corrigir ou acrescentar informações da ficha social dos pacientes.

- Cadastro de médicos: permite cadastrar, alterar ou excluir informações dos médicos cadastrados no sistema.

. Cadastro das clínicas: permite cadastrar, alterar ou excluir informações das clínicas cadastradas no sistema.

. Cadastro das unidades: permite cadastrar, alterar ou excluir informações das unidades médicas cadastradas no sistema.

. Cadastro de assistentes sociais: permite cadastrar, alterar ou excluir informações das assistentes sociais cadastradas no sistema.

- Relatório de produtividade hospitalar: permite cálculo semanal, mensal ou anual, da média de permanência, taxa de ocupação, pacientes-dia e leitos-dia de cada unidade hospitalar.

Para a elaboração do protótipo do sistema algumas características metodológicas foram adotadas, como, por exemplo, preservação dos documentos atuais do hospital, interface fiel à realidade e a produção de relatórios baseados na prática cotidiana. Diversas entrevistas e reuniões foram realizadas, tanto com o pessoal administrativo como com outros profissionais de saúde, sobretudo da área de enfermagem.

Embora as assistentes sociais do setor pudessem ser as mais beneficiadas com o volume de informações produzidas pelo sistema, elas reagiram de forma negativa, tentando até boicotar informações que delas dependiam. Ainda que as informações contidas no prontuário não sejam propriedade deste ou daquele profissional, as assistentes sociais se mobilizaram para que os dados da ficha social não fossem capturados pelo sistema.

É sabido que a tecnologia traz mudanças e ameaças, sobretudo para profissionais menos familiarizados com os avanços tecnológicos. É aceitável reações dessa natureza e é preciso estudá-las conjuntamente, de modo que a tecnologia não seja ameaça, mas ferramenta que venha auxiliar os profissionais a desempenharem as suas atividades com mais eficiência. A tecnologia deve ser desenvolvida para atender, de forma satisfatória, às necessidades do usuário e não tornar-se fator causador de ameaças e sofrimentos.

Talvez a falta de envolvimento dos profissionais do serviço social com o desenvolvimento do sistema tenha sido a causa principal das resistências ao funcionamento 
do sistema. Essa experiência demonstrou que um sistema de informação não deve ser considerado artefato puramente técnico, mas um sistema social, em que as pessoas são mais importantes do que a tecnologia.

\section{Conclusão}

Os resultados da automação do prontuário médico parecem ser promissores para a melhoria da qualidade do tratamento de saúde, redução de custos e avanço do conhecimento.

O desenho de um sistema de informação hospitalar requer considerações complexas, mas todo o esforço deve ser feito para vencer barreiras de ordem legal, organizacional e tecnológica. A gerência da complexidade da medicina moderna exige a automação dos registros médicos como tecnologia essencial aos cuidados médicos dos dias de hoje e do futuro.

Nos últimos anos começou a surgir nova geração de sistemas de informações hospitalares, priorizando a integração de informações clínicas e administrativas como a chave do seu sucesso. $\mathrm{O}$ avanço da tecnologia da informação no setor saúde em geral, e hospitalar em particular, exige uma educação paralela dos profissionais de saúde e um estudo destinado a melhorar as práticas de registro, indispensáveis à melhoria da qualidade do tratamento médico.

O sistema de registro de pacientes descrito neste trabalho, desenvolvido como o módulo básico e inicial de um sistema de informação hospitalar, foi um experimento apropriado para se avaliar a importância e complexidade do desenvolvimento ou desenho de um sistema de informação.

Assim, a importância da tecnologia da informação na área de saúde precisa ser mais pesquisada e discutida no nosso país, de modo que critérios de padronização e outros elementos sejam mais bem definidos, principalmente pelos profissionais de saúde. 


\section{Referências Bibliográficas}

BALL, M. J. et al.

Status and progress of hospital information system (HIS).

International Journal of Biomedical Computer, v. 29, p. 161-148, 1991.

BRAA, J.

Community-based participatory design in the third world. In: PARTICIPATORY DESIGN CONFERENCE (1996:

Cambridge). Proceedings... Cambridge: [s.n.], 1996.

BRUNUM, J. F.

The misinformation era : the fall of the medical record. Annals of Internal Medicine, v. 110 , p. 482-484, 1989.

\section{CHU, S.}

Clinical information systems : a fourth generation. Nursing Management, v. 24, n. 10, p. 59-60, 1993.

DAVIS, G. B. et al.

Diagnosis of an information system failure. Information and Management, v. 23, p. 293-318, 1992.

DICK, R. S.

The computer-based patient record : an essential technology for health care. Washington, DC: National Academy Press, 1991.

FRIEDMAN, B. A.;

MARTIN, J. B.

Hospital information system : the physician role. Journal of the American Medical Association, v. 257, n. 13, p. 1792, 1997.

GAO.

Medical ADP systems: automated medical record hold promise to improve patient care. Washington, DC : [s.n.], 1991.

HIRSHEY, C. O. et al.

The medical practice environment: internists' view of the future. Archives of Internal Medicine, v. 149, p. 1745-1749, 1989.

LINDBERG, D. A. B.;

HUMPHREYS, B. L.

Computers in medicine. Journal of the American Medical Association, v. 272, n. 231, p. 1667-1668, 1995.

MARKUS, M. L.

Power, politics and MIS implementation. Communication of the ACM, v. 26, n. 6, p. 430-444, 1983. 
MEGARGLE, R.

Role of ASTM in computer information standards for medicine. Topics in Health Record Management, v. 11, n. 4, p. 17-26, 1991.

POLLALIS, U. A.;

FRIEZE, I. H.

A new look at critical success factor in IT. Information Strategy, 1993.

PORIES, W. J.

Is medical record dangerous to our health? North Carolina Medical Journal, v. 51, p. 47-55, 1990.

RODRIGUES, R. J.

Informação e o administrador de saúde. São Paulo : Pioneria, 1987.

RODRIGUES FILHO, J.

Automação do arquivo médico.
Suprimentos e Serviços

Hospitalares, ano 1, n. 8, nov. 1995.

O paradigma interpretativo na pesquisa e desenvolvimento de sistemas de informação. In: 16TH ANNUAL CONFERENCE OF THEBALAS (1999: New Orleans). Proceedings... New Orleans: BALAS, 1999.

STEAD, W. W.

A quarter-century of computer-based medical records. [S.1.] : MD Computing, 1989.

THOMAS, R. et al.

Access to health information and support : a public highway or a private road? Journal of the American Medical Association, v. 280, n. 15, p. 1371-1374, 1998. 